\title{
Expansão urbana e distribuição espacial da malária no município de Manaus, Estado do Amazonas
}

\author{
Urban expansion and spatial distribution of malaria in the \\ municipality of Manaus, State of Amazonas
}

\author{
Maria das Graças Gomes Saraiva ${ }^{1,2}$, Raul Diniz Souza Amorim², \\ Marco Antônio Sabóia Moura ${ }^{2}$, Flor Ernestina Martinez-Espinosa ${ }^{1,2}$ \\ e Maria das Graças Vale Barbosa ${ }^{1,2}$
}

\begin{abstract}
RESUMO
No município de Manaus, a intensificação do processo migratório, aliada a precária vigilância epidemiológica e entomológica, resultou na reintrodução da transmissão de malária no perímetro urbano, zona Leste, em julho de 1988, após 13 anos sem registro de autoctonia. Este estudo descreve sobre a situação epidemiológica da malária e áreas que sofreram ações antrópicas (desmatamento, assentamentos humanos, atividades de piscicultura, etc.) em Manaus, no período entre 1986 e 2005. Nesse Município, o incremento populacional em 2005 atingiu 105,2\%, em relação a 1986, resultado de ocupação dos espaços (invasões e conjuntos habitacionais). A partir de 2003, a doença teve incremento acima 2.000\% em relação a 1986. Nessas áreas ocorreu aumento da incidência da doença. 0 índice parasitário anual no Município oscilou de baixo a médio risco, e entre as zonas urbanas, variou de sem risco a alto risco. As zonas Leste, Oeste e Norte onde ainda existem áreas com características rurais apresentaram maior receptividade e vulnerabilidade de transmissão.
\end{abstract}

Palavras-chaves: Malária. Ações antrópicas. Ocupação dos espaços.

\begin{abstract}
In the municipality of Manaus, intensification of the migratory process, along with precarious epidemiological and entomological surveillance, resulted in reintroduction of malaria transmission on the urban perimeter (in the eastern zone), in July 1988, after 13 years without any records of autochthonous disease. This study reports on the epidemiological situation relating to malaria and to the areas that were subjected to human actions (deforestation, human settlement, fish-rearing activity, etc) in Manaus, between 1986 and 2005. In this municipality, the population increase from 1986 to 2005 was $105.2 \%$. This resulted from occupation of space, in the form of invasions and housing projects. From 2003 , the increase in relation to 1986 was more than $2,000 \%$. In these areas, there were increases in disease incidence. The annual parasitic index in the municipality ranged from low to medium risk and, between urban zones, it ranged from no risk to high risk. In the eastern, western and northern zones, which still contain areas with agricultural characteristics, there was greater receptivity and vulnerability to transmission.
\end{abstract}

Key-words: Malaria. Human actions. Occupation of spaces.

A malária é doença infecciosa, febril, aguda, parasitária, sistêmica, não contagiosa e de transmissão vetorial, causada por protozoários que infectam alternadamente um hospedeiro vertebrado e um invertebrado ${ }^{4} 1518$. Seus transmissores são mosquitos do gênero Anopheles, sendo o Anopheles (Nyssorhynchus) darlingi, a principal espécie vetora no Brasil ${ }^{8}$. Nas Américas, três espécies de protozoários causam a malária em seres humanos: Plasmodium vivax, Plasmodium falciparum

\footnotetext{
1. Pós-graduação em Medicina Tropical da Universidade do Estado do Amazonas, Manaus, AM. 2. Gerências de Entomologia, Informática, Departamento de Epidemiologia e Saúde Pública, Fundação de Medicina Tropical do Amazonas, Manaus, AM.

Endereço para correspondência: Eng M.Sc. Maria das Graças Gomes Saraiva. Dept ${ }^{\circ}$ de Epidemiologia e Saúde Pública/ FMT-AM. Av. Pedro Teixeira 25, Dom Pedro, 69 040-000 Manaus, AM

Tel: 5592 2127-3438

e-mail: gracasaraiva@fmt.am.gov.br

Recebido para publicação em 07/05/2009

Aceito em 29/09/2009
}

e Plasmodium malariae. Uma quarta espécie, o Plasmodium ovale, pode ser encontrada no continente africano e sudoeste $\operatorname{asiático~}^{10}$.

No Brasil, cerca de 99,5\% dos casos ocorre na Amazônia Legal, considerada região endêmica. Nos últimos anos, Manaus e Porto Velho apresentaram extensas áreas de aglomerados urbanos em regiões periféricas, importantes locais de infecção devido a intenso fluxo de pessoas procedentes de outros municípios em busca de oportunidades de trabalho ou necessidades comerciais. Esses municípios concentraram 26,9\% e 22,9\% dos casos de malária da Região Amazônica nos anos de 2003 e 2004, respectivamente ${ }^{17}$.

0 risco de contrair a doença não é uniforme, sendo medido por índice parasitário anual (IPA) que classifica as áreas de transmissão em alto risco-IPA maior que 49,9 casos de malária por mil habitantes; médio risco-IPA entre 10 e 49,9 casos/1.000 habitantes, baixo risco-IPA de 0,1 a 9,9 casos/1.000 habitantes, e zero-sem risco ${ }^{16}$. 
As áreas de alto risco têm como características epidemiológicas floresta tropical úmida que favorece a transmissão perene e focalmente intensa, principalmente em grupos de trabalhadores expostos, alta prevalência de Plasmodium falciparum geralmente resistente a antimaláricos e populações migrantes com escassa imunidade, expostas às altas densidades de Anopheles darlingi, dentro e fora de moradias precárias que não oferecem proteção. As áreas de médio risco correspondem à floresta menos densa por ocupação humana mais antiga; população residente com maior imunidade; migração localizada especialmente e de áreas rurais a urbanas; habitações mais protetoras com as densidades menores de Anopheles darlingi, localizadas principalmente nas margens dos grandes rios; transmissão estacional com reativação focal; predomínio de Plasmodium vivax; infra-estrutura social mais desenvolvida, com maiores facilidades de comunicaçã $0^{9}$.

As áreas de baixo risco são aquelas de malária instável onde a transmissão foi interrompida nas décadas de 1960-1970. Porém, conservam o potencial malarígeno que dá origem à transmissão eventual em áreas restritas, predomínio de vetores secundários, populações estáveis, com infra-estrutura social bem desenvolvida. As áreas sem risco são aquelas com ausência de fatores epidemiológicos necessários para a transmissão de malária9.

Os surtos da malária surgem em função da aglomeração de indivíduos em habitações precárias ou simples abrigos, onde estão reunidos os elos da cadeia epidemiológica da malária, ou seja, os portadores do plasmódio, vetores e indivíduos suscetíveis ${ }^{1111}$. As coleções hídricas artificiais constituídas de barragens e tanques de piscicultura têm se configurado como importantes criadouros permanentes de mosquito transmissor da malária, devendo ser considerados entre os fatores ambientais de riscos passíveis de intervenções ${ }^{22}$.

No Amazonas, entre 1960 e 1980, a malária apresentou baixos níveis endêmicos, com ocorrência de epidemias em poucos municípios, controladas em médio prazo ${ }^{14}$. Em Manaus, a incidência de malária é epidêmica, em decorrência da presença de grandes levas de migrantes, que se deslocaram para a periferia da cidade, favorecendo condições de multiplicação dos criadouros de anofelinos, instalando o ciclo de transmissã $0^{5}$. 0 desmatamento e a ocupação de áreas, antes cobertas por mata provida de mananciais, agora com assentamentos humanos, de forma desordenada (invasões) ou programada (conjuntos habitacionais) e estabelecimento de práticas laborais (atividades de piscicultura), têm sido fatores determinantes para a reintrodução e a permanência da malária, principalmente em áreas urbanas da cidade. Portanto, este trabalho teve o objetivo de descrever a incidência e a distribuição geográfica dos casos autóctones de malária em áreas do município de Manaus que sofreram ações antrópicas, no período entre 1986 e 2005.

\section{MATERIAL E MÉTODOS}

0 trabalho foi realizado através de estudo ecológico, descritivo, por meio de levantamento de dados de fontes secundárias.
0 município de Manaus abrange uma área com $11.458,5 \mathrm{~km}^{2}$ de extensão, dividido em áreas urbanas, peri-urbana e rural, localizadas em margens de rios, igarapés e as estradas BR - 174, que liga Manaus a Boa Vista/Roraima e AM - 010, rodovia que liga Manaus ao município de Itacoatiara, no Estado do Amazonas.

Foram levantadas informações criando-se uma série histórica incluindo-se dados epidemiológicos de todos os doentes febris registrados para diagnóstico de malária, que compareceram aos serviços de saúde de Manaus, no período de 1986 a 2005. Também, foram plotadas em figuras a expansão urbana do Município, e descrito os programas para o controle da doença.

A verificação do IPA foi a partir das bases de dados SISMAL (Sistema Informatizado de Malária) e SIVEP-Malária (Sistema de Informações de Vigilância Epidemiológica-Malária); obtenção do registro de casos de malária por região de Manaus foi possível através do SIVEP-Malária, referindo-se ao período de 2001 a 2005.

Foram feitas buscas ativas sobre informações de relatórios técnicos da Fundação Nacional de Saúde (FUNASA), Secretaria de Vigilância em Saúde (SVS) do Ministério da Saúde (MS) e da Fundação de Medicina Tropical do Amazonas (FMT-AM). As informações foram analisadas de acordo com mês, ano, forma parasitária, distribuição dos casos por localidades e regiões, índice parasitário anual (IPA), índice de Plasmodium falciparum anual (IFA), índice anual de exames de sangue (IAES) e índice de lâminas positivas (ILP), calculados a partir das seguintes equações:

ILP: índice de lâminas positivas (\%)

$$
I L P=\frac{N E P}{N_{p}} .100[\text { eq.01] }
$$

Onde:

NEP: número de exames positivos no período;

$\mathrm{N}_{\mathrm{p}}$ : número de exames no mesmo período.

IFA: índice de Plasmodium falciparum anual (\%)

$$
I F A=\frac{N E F+N M}{N E P} .100[\text { eq.02] }
$$

Onde:

NEF: número de casos de Plasmodium falciparum;

NM: número de malária mista (o número de casos de Plasmodium falciparum + o número de casos de Plasmodium vivax). 
IPA: índice parasitário anual - incidência (casos por 1.000 habitantes (\%o)

$$
\mathrm{IPA}=\frac{N E P}{P} .1000[\mathrm{eq} .03]
$$

Onde:

P: número da população no período.

IAES: índice anual de exames de sangue no período (\%)

$$
I A E S=\frac{N_{P}}{P} .100[\mathrm{eq} .04]
$$

Para a descrição da incidência da doença no período estudado, foi feito um levantamento anual da estimativa populacional de Manaus, utilizando-se fonte de dados do Instituto Brasileiro de Geografia e Estatística (IBGE), com vistas ao cálculo do IPA e do IAES. Para a descrição da distribuição dos casos nas diferentes áreas descritas, foram utilizadas informações do Instituto Municipal de Planejamento Urbano (IMPLURB), sobre a expansão urbana de Manaus no período estudado.

Para se obter o percentual de participação das zonas Norte, Leste e Oeste utilizou-se as seguintes equações:

$\mathrm{AC}_{\mathrm{u}}$ : número de casos acumulados (\%)

$$
A C_{u}=\frac{\sum C_{u}}{T C_{u}+T C_{r}} .100[\text { eq.05] }
$$

Onde:

$\mathrm{C}_{\mathrm{u}}$ : número de casos das zonas;

$\mathrm{TC}_{\mathrm{u}}$ : total de casos da área urbana;

$\mathrm{TC}_{\mathrm{r}}$ : total de casos da área rural.

$\mathrm{Z}_{\mathrm{n}}$ : número de casos por zona.

$$
C_{u}=\sum Z_{1}+Z_{2}+Z_{3} \ldots Z_{n}[\text { eq.06] }
$$

$\mathrm{PZ}_{\mathrm{A}}$ : participação das zonas na área urbana (\%).

$$
P Z_{A}=\frac{\sum C_{u}}{T C_{u}}[\text { eq.07] }
$$

0 número de casos aplicados na equação foram: 141.180 que correspondem ao total de casos da área urbana, e 140.306 que correspondem ao valor acumulado das citadas. E o total geral (zonas urbanas e rurais) 211.313.

Para a descrição das medidas de combate à doença, foram incluídas informações sobre a infestação vetorial durante 0 período estudado adquiridas a partir de relatórios da FUNASA, da Secretaria Estadual da Saúde - Fundação de Vigilância em Saúde (FVS), da Gerência de Entomologia da FMT-AM e de buscas sobre publicações em congressos e revistas.

Foi criado um banco de dados no software Epi-Info 2003 com os dados revisados para a detecção de erros lógicos e inconsistências nos registros. Para posterior, tabulação e análise das informações. Para a distribuição dos casos por localidades e por regiões, foi utilizado o programa ArcGIS®.

\section{RESULTADOS}

Descrição epidemiológica da malária em Manaus no período entre 1986 e 2005. Durante o período do estudo, observou-se em 2005 um incremento populacional progressivo atingindo 105,2\% (1.644.690 habitantes) em relação a 1986 (801.708 habitantes) (Figura 1).

Entre 1986 e 2005, foram realizados 2.006.668 exames hemoscópicos, dos quais 407.435 (20,3\%) foram positivos para malária. O menor registro ocorreu em 1986 (2.463 casos), e o maior em 2003 (69.306). 0 índice parasitário anual foi maior em 2003 (45,4 casos/1.000 habitantes - médio risco) e menor em 1987 (2,9 casos/1.000 hab - baixo risco). 0 índice de lâminas positivas (ILP) foi maior em 1991 (43,5\%), e menor em 2001 (6\%). 0 índice de Plasmodium falciparum anual (IFA) foi maior em 1986 (40,8\%) e menor em 2003 (6,5\%). Dentre os exames positivos, 339.026 representando $83,2 \%$ foram por Plasmodium vivax, 65.452 (16,1\%) por Plasmodium falciparum e $2.957(0,7 \%)$ por Plasmodium vivax + Plasmodium falciparum (infecção mista). Em relação à malária por Plasmodium vivax, registrou-se maior número de positivos em 2003 (64.770) e menores em 1986, com 1.459. Na malária por Plasmodium falciparum, o maior registro do número de casos ocorreu em 2005 com 14.397 e menor em 1987 com 863 casos (Tabela 1).

Descrição da malária por região do município de Manaus entre 1986 e 2005. Área urbana: entre 2001 e 2005, a área urbana de Manaus foi considerada uma área de baixo risco, com variação do IPA em 2001 e 2002 de 2,9 casos/1.000 habitantes e 8,3 casos $/ 1.000$ hab, respectivamente. No período de 2003 a 2005, ocorreu variação entre 22,3 e 32,7 casos/1.000 habitantes, passando a situação de médio risco (Tabela 2). Área rural: os maiores índices parasitários foram atribuídos à área rural, onde o IPA aumentou gradativamente ano a ano, passando de 129,1 casos/mil habitantes em 2001 para 1.659,7 casos /1.000 hab. em 2005, representando alto risco (Tabela 2).

Distribuição do IPA por zonas geográficas. Em 2001, as zonas Centro Oeste e Sul foram classificadas como áreas sem risco e as outras zonas como de baixo risco. Entretanto, em 2002, as zonas Leste e Oeste foram classificadas como áreas de médio risco e passaram a ser consideradas como áreas de alto risco em 2003, sendo que a zona Oeste se manteve como área de alto risco até 2005 (Figura 2). Distribuição por zonas: de maneira geral, de 2001 a 2005, nos registros da distribuição geográfica de 


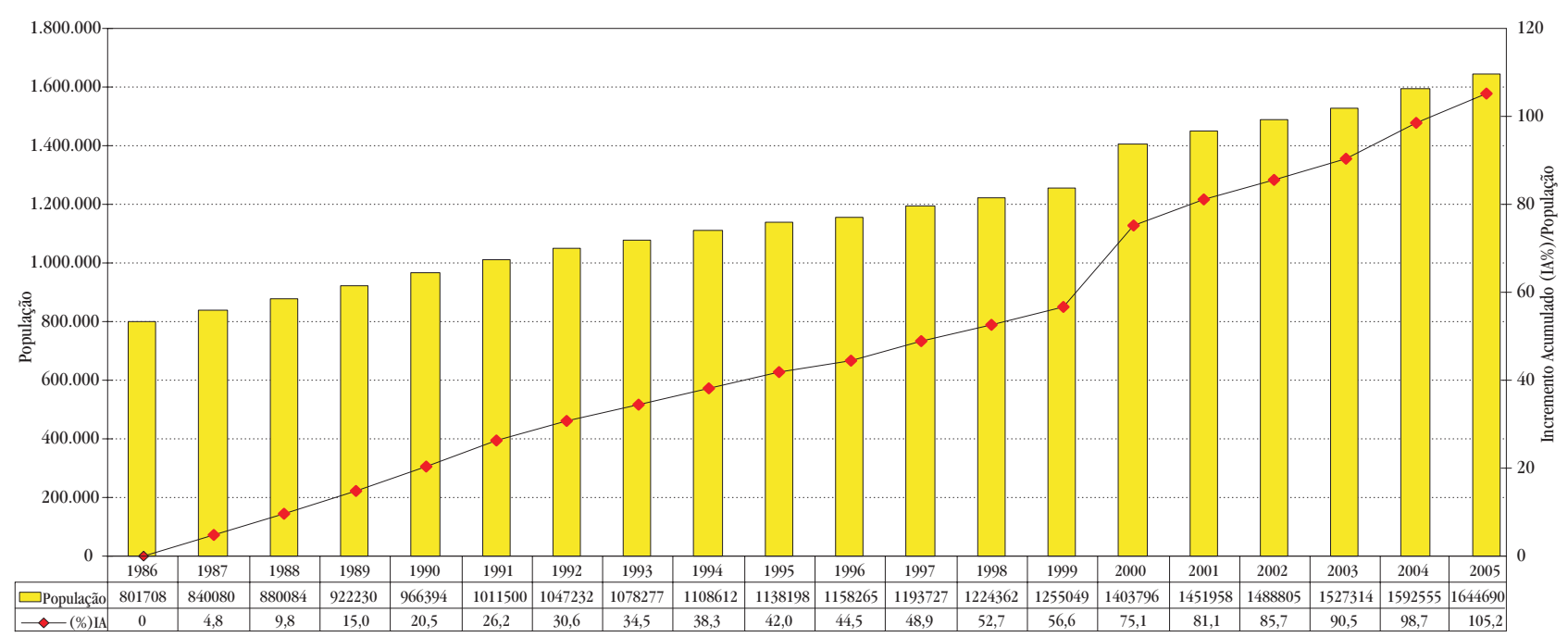

FIGURA 1

Distribuição da população do município de Manaus e incremento acumulado populacional entre 1986 e 2005.

TABELA 1

Distribuição da população, exames realizados, exames positivos e índices da malária no município de Manaus entre 1986 e 2005.

\begin{tabular}{|c|c|c|c|c|c|c|c|c|c|c|}
\hline Ano & População & Exames & Positivos & $\mathbf{F}$ & $\mathbf{V}$ & $\mathbf{F}+\mathbf{V}$ & ILP (\%) & IFA (\%) & IPA (\%o) & IAES (\%) \\
\hline 1986 & 801.708 & 22.806 & 2.463 & 956 & 1.459 & 48 & 10,8 & 40,8 & 3,1 & 2,8 \\
\hline 1987 & 840.080 & 10.885 & 2.472 & 863 & 1.555 & 54 & 22,7 & 37,1 & 2,9 & 1,3 \\
\hline 1988 & 880.084 & 20.455 & 3.843 & 1.199 & 2.591 & 53 & 18,8 & 32,6 & 4,4 & 2,3 \\
\hline 1989 & 922.230 & 33.487 & 9.180 & 1.944 & 7.170 & 66 & 27,4 & 21,9 & 10,0 & 3,6 \\
\hline 1990 & 966.394 & 22.380 & 6.386 & 1.347 & 4.998 & 41 & 28,5 & 21,7 & 6,6 & 2,3 \\
\hline 1991 & 1.011 .500 & 31.963 & 13.901 & 1.068 & 12.796 & 37 & 43,5 & 7,9 & 13,7 & 3,2 \\
\hline 1992 & 1.047 .232 & 31.563 & 11.712 & 989 & 10.712 & 11 & 37,1 & 8,5 & 11,2 & 3,0 \\
\hline 1993 & 1.078 .277 & 61.408 & 23.189 & 5.605 & 17.471 & 113 & 37,8 & 24,7 & 21,5 & 5,7 \\
\hline 1994 & 1.108 .612 & 52.182 & 17.224 & 3.012 & 14.084 & 128 & 33,0 & 18,2 & 15,5 & 4,7 \\
\hline 1995 & 1.138 .198 & 47.590 & 11.828 & 2.107 & 9.449 & 272 & 24,9 & 20,1 & 10,4 & 4,2 \\
\hline 1996 & 1.158 .265 & 55.786 & 12.593 & 2.187 & 10.202 & 204 & 22,6 & 19,0 & 10,9 & 4,8 \\
\hline 1997 & 1.193 .727 & 72.361 & 21.234 & 3.496 & 17.626 & 112 & 29,3 & 17,0 & 17,8 & 6,1 \\
\hline 1998 & 1.224 .362 & 76.577 & 17.995 & 2.472 & 15.439 & 84 & 23,5 & 14,2 & 14,7 & 6,3 \\
\hline 1999 & 1.255 .049 & 104.722 & 23.861 & 4.002 & 19.723 & 136 & 22,8 & 17,3 & 19,0 & 8,3 \\
\hline 2000 & 1.403 .796 & 107.739 & 18.241 & 3.314 & 14.646 & 281 & 16,9 & 19,7 & 13,0 & 7,7 \\
\hline 2001 & 1.451 .958 & 97.207 & 5.808 & 1.002 & 4.758 & 48 & 6,0 & 18,1 & 4,0 & 6,7 \\
\hline 2002 & 1.488 .805 & 137.545 & 15.865 & 1.404 & 14.411 & 50 & 11,5 & 9,2 & 10,7 & 9,2 \\
\hline 2003 & 1.527 .314 & 315.687 & 69.306 & 4.284 & 64.770 & 252 & 22,0 & 6,5 & 45,4 & 20,7 \\
\hline 2004 & 1.592 .555 & 332.186 & 55.940 & 9.804 & 45.770 & 366 & 16,8 & 18,2 & 35,1 & 20,9 \\
\hline 2005 & 1.644 .690 & 372.139 & 64.394 & 14.397 & 49.396 & 601 & 17,3 & 23,3 & 39,2 & 22,6 \\
\hline Total & & 2.006 .668 & 407.435 & 65.452 & 339.026 & 2.957 & & & & \\
\hline
\end{tabular}

Fonte: População dados do IBGE/FUNASA/SISMAL/SIVEP-Malária/SVS/MS

F: malária por Plasmodium falciparum, V: malária por Plasmodium vivax, F+V: malária por Plasmodium falciparum + Plasmodium vivax (mista), ILP: índice de lâminas positivas (\%), IFA: índice de Plasmodium falciparum anual $=\%\{\mathrm{~F}+(\mathrm{F}+\mathrm{V})\}$, IPA: índice parasitário anual - incidência (casos por 1.000 habitantes (\%), IAES: índice anual de exames de sangue (\% habitantes)

casos da malária envolvendo localidades/bairros/zonas urbanas, observou-se registro de casos de malária em todos os bairros da zona Norte; na Leste, apenas no Coroado não houve registro de casos; na Oeste, registraram-se casos no Tarumã e na Ponta Negra; na Centro Sul foi registrado casos em Flores, e na zona Centro Oeste registro de casos somente em Redenção.

Casos autóctones de malária por região do município de Manaus de 2001 e 2005. Entre 2001 e 2005, foram registrados 211.313 casos autóctones de malária, nas zonas de Manaus (urbana e rural). Desses, 141.180 (66,8\%) casos ocorreram na área urbana e $70.133(33,2 \%)$ casos na área rural. Na área urbana, as zonas Norte, Leste e Oeste juntas, produziram 66,4\% do total de casos e 99,4\% do total de casos da área urbana. Do total de casos registrados nas áreas urbanas e rurais, teve-se por Plasmodium falciparum + infecção mista 32.208 (IFA = 15,2\%) casos, com maior número na zona Oeste (Tabela 2). 
TABELA 2

Número de casos de malária, índice parasitário anual e índice de Plasmodium falciparum por região de Manaus entre 2001 e 2005 .

\begin{tabular}{|c|c|c|c|c|c|c|}
\hline \multirow[b]{2}{*}{ Região/Zonas } & \multirow[b]{2}{*}{ Especificação } & \multicolumn{5}{|c|}{ Anos } \\
\hline & & 2001 & 2002 & 2003 & 2004 & 2005 \\
\hline \multirow[t]{4}{*}{ Norte } & casos & 268 & 2.578 & 10.297 & 6.202 & 7.428 \\
\hline & população & 312.376 & 344.286 & 379.781 & 428.891 & 480.710 \\
\hline & IPA & 0,9 & 7,5 & 27,1 & 14,5 & 15,5 \\
\hline & IFA & 12,3 & 5,9 & 3,8 & 10,7 & 18,1 \\
\hline \multirow[t]{4}{*}{ Leste } & casos & 2.635 & 6.886 & 21.420 & 17.029 & 17.518 \\
\hline & população & 359.975 & 377.503 & 394.197 & 417.071 & 435.729 \\
\hline & IPA & 7,3 & 18,2 & 54,3 & 40,8 & 40,2 \\
\hline & IFA & 29,0 & 10,4 & 7,0 & 18,4 & 21,8 \\
\hline \multirow[t]{4}{*}{ Oeste } & casos & 1.307 & 2.802 & 17.579 & 11.754 & 14.603 \\
\hline & população & 210.973 & 206.234 & 202.140 & 201.325 & 199.884 \\
\hline & IPA & 6,2 & 13,6 & 87,0 & 58,4 & 73,1 \\
\hline & IFA & 5,1 & 13,2 & 6,3 & 17,3 & 22,4 \\
\hline \multirow[t]{4}{*}{ Centro Oeste } & casos & 0 & 0 & 0 & 0 & 112 \\
\hline & população & 140.350 & 137.995 & 134.852 & 132.809 & 128.094 \\
\hline & IPA & 0,0 & 0,0 & 0,0 & 0,0 & 0,9 \\
\hline & IFA & 0,0 & 0,0 & 0,0 & 0,0 & 14,3 \\
\hline \multirow[t]{4}{*}{ Centro Sul } & casos & 28 & 28 & 264 & 234 & 208 \\
\hline & população & 127.012 & 128.641 & 130.178 & 133.015 & 133.253 \\
\hline & IPA & 0,2 & 0,2 & 2,0 & 1,8 & 1,6 \\
\hline & IFA & 21,4 & 7,1 & 6,1 & 10,7 & 19,8 \\
\hline \multirow[t]{4}{*}{ Sul } & casos & 0 & 0 & 0 & 0 & 0 \\
\hline & população & 289.111 & 281.300 & 272.640 & 265.216 & 252.243 \\
\hline & IPA & 0,0 & 0,0 & 0,0 & 0,0 & 0,0 \\
\hline & IFA & 0,0 & 0,0 & 0,0 & 0,0 & 0,0 \\
\hline \multirow[t]{4}{*}{ Subtotal } & casos & 4.238 & 12.294 & 49.560 & 35.219 & 39.869 \\
\hline & população & 1.439 .797 & 1.475 .959 & 1.513 .788 & 1.578 .327 & 1.629 .913 \\
\hline & IPA & 2,9 & 8,3 & 32,7 & 22,3 & 24,5 \\
\hline & IFA & 20,6 & 10,1 & 6,1 & 16,6 & 21,3 \\
\hline \multirow[t]{4}{*}{ Rural } & casos & 1.570 & 3.571 & 19.746 & 20.721 & 24.525 \\
\hline & população & 12.158 & 12.846 & 13.526 & 14.228 & 14.777 \\
\hline & IPA & 129,1 & 278,0 & 1459,9 & 1456,4 & 1659,7 \\
\hline & IFA & 11,4 & 6,0 & 7,7 & 20,8 & 26,5 \\
\hline \multirow[t]{4}{*}{ Total } & casos & 5.808 & 15.865 & 69.306 & 55.940 & 64.394 \\
\hline & população & 1.451 .955 & 1.488 .805 & 1.527 .314 & 1.592 .555 & 1.644 .690 \\
\hline & IPA & 4,0 & 10,7 & 45,4 & 35,1 & 39,2 \\
\hline & IFA & 18,1 & 9,2 & 6,5 & 18,2 & 23,3 \\
\hline
\end{tabular}

Fonte: IBGE/SEMSA/SISMAL/SIVEP-Malária/SVS/MS

IPA: índice parasitário anual - íncidência (casos por 1.000 habitantes (\%o)), IFA: índice de Plasmodium falciparum anual $=\%\{\mathrm{~F}+(\mathrm{F}+\mathrm{V})\}$

Verificou-se que $1.535(0,7 \%)$ casos de malária foram referenciados como de áreas urbanas da cidade de Manaus, dentre os quais, Adrianópolis, Alvorada, Cachoerinha, Centro, Dom Pedro, Morro da Liberdade, Petrópolis, Praça 14 de Janeiro, Santo Antônio, Santo Agostinho, São Francisco, São Geraldo, São Raimundo e São Lázaro, locais onde as atuais condições ambientais demonstram ser impróprias ao desenvolvimento de anofelino.

Registrou-se 28.625 (13,5\%) casos de malária em áreas rurais na estrada Rodovia BR - 174, e 21.497(10,2\%) casos da doença em áreas rurais na estrada AM - 010, em locais do Rio Negro, teve-se o registro de 12.122 (5,7\%) casos e no Rio Amazonas 19.969 (9,4\%) casos desse agravo.

Consequências da expansão da área urbana de Manaus. A partir da metade da década de 1980, a área urbana de Manaus sofreu ação antrópica, resultando em seu desmatamento e consequentemente na alteração ambiental. Áreas antes cobertas por vegetação foram gradativamente sendo desmatadas dando espaço à expansão urbana da Cidade, restando pouca vegetação (Figura 3). 


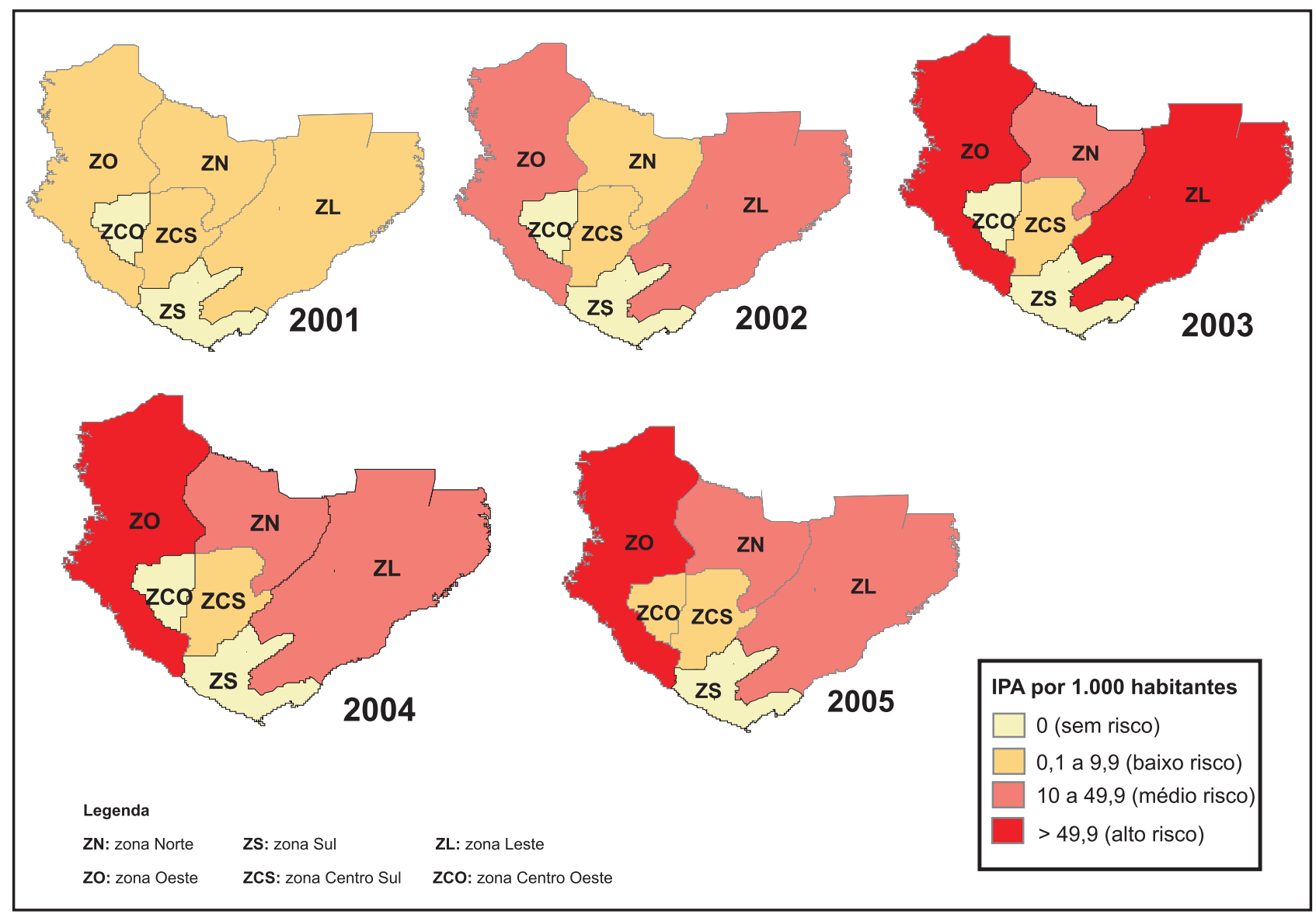

FIGURA 2

Representação do índice parasitário anual por zonas urbanas de Manaus de 2001 a 2005.

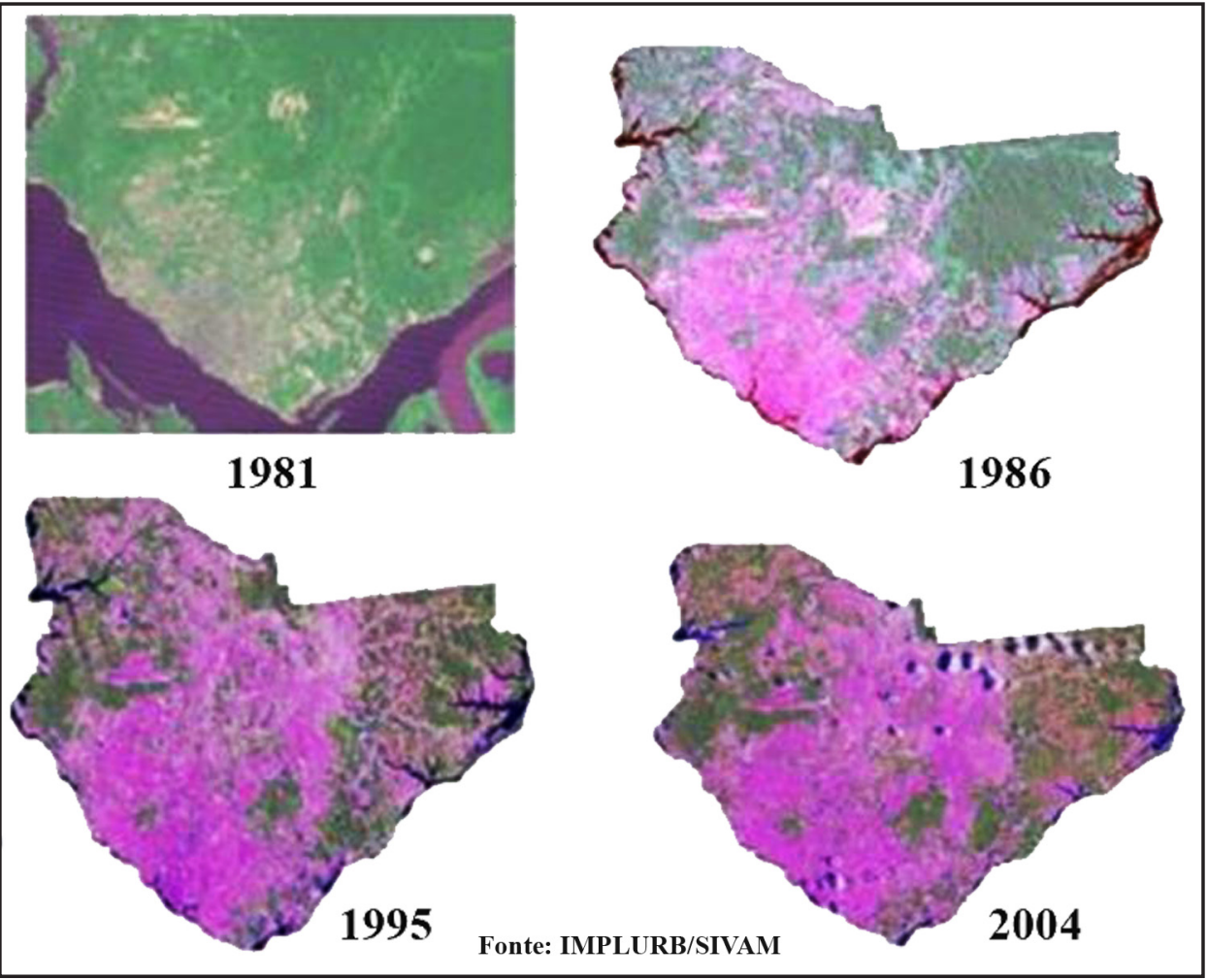

FIGURA 3

Imagens landsat da expansão da zona urbana de Manaus nas décadas de 1981, 1986, 1995 e 2004. 
Expansão por zonas consideradas endêmicas. As zonas Leste, Norte e Oeste da Cidade de Manaus, entre 1986 e 2004, foram áreas que mais sofreram desmatamento, mas, ainda possuem vasta cobertura vegetal, com possibilidade de expansão representando para a população risco de transmissão e manutenção da malária (Figura 3).

Planos de ação para controle da malária. No levantamento de planos de ação para o controle da malária, durante o período do estudo, observou-se que foram estabelecidos dez planos para o município de Manaus. As propostas de ações básicas para interromper a transmissão de malária envolviam principalmente combate ao parasito e ao vetor através de notificação de casos, estruturação de laboratórios para o diagnóstico, e pesquisa larvária com captura de adultos.

\section{DISCUSSÃO}

Descrição epidemiológica da malária em Manaus entre 1986 e 2005. 0 incremento populacional progressivo na Cidade de Manaus, nos últimos 20 anos contribuiu para a expansão e a ocupação desordenada (invasões) ou programada dos espaços (grandes conjuntos habitacionais). Neste período, houve 0 monitoramento da doença, através de exames hemoscópicos dos doentes febris; entretanto, é possível que o suporte da vigilância epidemiológica e entomológica não tenha conseguido acompanhar o crescimento demográfico, observado nas flutuações do número acumulado de casos de malária e consequentemente do índice parasitário anual, mantendo na área periférica de Manaus, o status de baixo, médio e alto risco, expondo a população ao risco de contrair a doença.

0 acometimento foi maior por Plasmodium vivax e em 2005, notou-se aumento das infecções por Plamodium falciparum. Nas regiões endêmicas, a doença por Plasmodium falciparum é um problema de saúde pública, por ser a principal espécie causadora de malária grave e pelas altas taxas de morbidade e mortalidade, principalmente, quando se considera a infecção nas crianças. No entanto, na Amazônia Brasileira tem sido observado casos graves pelo Plasmodium vivax 3 .

Distribuição da malária por zonas geográficas de Manaus. As informações sobre a ocorrência da doença por bairros e localidades urbanas e rurais, foram limitadas, porque somente a partir de 2001, houve disponibilização dos dados nos sistemas informações. Num período de cinco anos, os maiores índices parasitários foram registrados na área rural. Provavelmente, por ser o local onde muitas pessoas passam seus fins de semana, feriados e férias, retornando infectados para área urbana, elevando a autoctonia dos casos e o valor do IPA, uma vez que tem baixa densidade populacional. 0 índice parasitário anual é o parâmetro para se estimar a possibilidade de ocorrência de casos de malária ${ }^{16}$.

Sobre a distribuição da doença nas áreas urbanas, na zona Sul, mais antiga da cidade houve notificação de autoctonia, entretanto, essa área não apresenta características ambientais que favoreçam a viabilidade de criadouros de anofelinos, sugerindo registro inadequado no sistema de informação. As zonas Leste, Oeste e Norte, a partir da década de noventa apresentaram maior receptividade e vulnerabilidade de transmissão confirmada pela concentração de 66,4\% dos casos registrados no Município, de 2001 a 2005, sendo consideradas as áreas mais importante dessa doença. No Amazonas, em 1990, três municípios, apresentavam IPA acima de 100 lâminas positivas por 1.000 habitantes 5 dentre eles Manaus, que em 1993 registrou o maior IPA dos últimos 30 anos $^{1}$.

Consequências da expansão da área urbana de Manaus. No período de 1986 a 2004, ocorreram desmatamentos e expansão urbana acentuada nas áreas de transmissão e manutenção da doença (zonas Oeste, Leste e Norte), cujas ocupações dos espaços contribuíram para um perfil epidemiológico epidêmico da malária em Manaus, principalmente porque a população da área rural abandona suas terras e procura emprego nas áreas urbanas, criando a instalação de favelas ${ }^{5}$, o que pode ser observado através do incremento populacional de Manaus, e da inclusão de novas áreas habitacionais, à expansão urbana programada (conjuntos habitacionais) e invasões, locais sem infra-estrutura que mantém a Cidade como área de transmissão desse agravo.

0 monitoramento dessas situações de risco pode ser realizado por meio de técnicas de sensoriamento remoto e sistemas de informações geográficas que podem auxiliar nas ações de controle da endemia, em regiões de evidente risco ambiental, onde ações específicas de proteção e assistência às populações mais vulneráveis podem ser priorizadas ${ }^{23}$.

É na forma da organização socioambiental que as doenças encontram espaços para ora emergirem, e ora ganharem novas faces $^{12}$. Em Manaus, nas áreas de risco, alguns fatores como o desmatamento, represamento de águas dos igarapés e atividades de piscicultura (tanques e barragens para criação de peixes) proporcionaram a formação de criadouros permanentes potenciais para 0 vetor ${ }^{22}$, viabilizando a permanência de reservatórios do Plasmodium. 0 Anopheles darlingi, mais importante vetor de malária na Amazônia, se alimenta tanto fora (exofagia) quanto no interior (endofagia) das casas, transmitindo a malária, mesmo em baixa densidade. É possível que esse comportamento do vetor seja um dos fatores contribuintes para dificultar o controle da doença em áreas de transmissão da malária em Manaus ${ }^{20}$. 0 s tipos de construções das casas, parcialmente ou sem paredes, dotadas de frestas no piso e abertura no telhado para facilitar a ventilação, favorecem a entrada do vetor no domicílio e contribuem para a ocorrência desse agravo $0^{14}$.

História do controle da malária em Manaus. A prática do homem na ocupação dos espaços aliadas as condições ambientais e climáticas favoráveis à produção da doença em Manaus, denota um grande desafio ao seu controle. Há relatos de que entre 1976 e junho de 1988, a cidade ficou sem ocorrência de casos autóctones, mas a intensificação do processo migratório, aliada à precária vigilância epidemiológica e entomológica, resultou na reintrodução da transmissão da malária no perímetro urbano, detectando-se em julho de 1988 o vetor e casos autóctones na zona Leste, bairros Tancredo Neves e São José Operário, o que não ocorria desde 1975. Nesse período, dez planos com boas 
propostas de monitoramento foram implantados ${ }^{13}$. Entretanto, a malária em Manaus teve momentos de flutuações, talvez devido à falta de continuidade das ações propostas nos planos e de avaliação dos impactos. Essa doença se mantém até os dias atuais como um sério problema de saúde pública, principalmente nas áreas urbanas da capital do Amazonas. Assim, para se conseguir redução do reservatório de Plasmodium, as medidas de controle para a eliminação de mosquitos infectados devem ser integradas ${ }^{21}$.

Essa proposta vem sendo desenvolvida pela Fundação de Vigilância em Saúde, recém criada no Amazonas, o que pode resultar em uma diminuição do registro da malária, colocando o índice parasitário anual em níveis aceitáveis para redução do risco de adoecer por esta doença nesta Região. A malária não confere imunidade permanente às suas vítimas, e um mesmo indivíduo pode ter dezenas de malárias e continuar vulnerável. A resposta imune protetora não é efetiva, sendo a imunidade adquirida com o aumento da exposição ao risco de infecção, pela conversão dessa doença potencialmente grave, ou seja, com alta densidade parasitária e sinais e sintomas importantes, para forma crônica benigna sem ou com poucos sintomas e pauciparasitêmica ${ }^{67}$.

\section{AGRADECIMENTOS}

Os autores agradecem a todos os colaboradores do Departamento de Epidemiologia e Saúde Pública/Fundação de Medicina Tropical do Amazonas e da Fundação de Vigilância em Saúde do Amazonas, pela contribuição na aquisição dos dados sobre a malária. Ao Dr. Antonio Geraldo Silva da Rocha, Gerência de Informação - GIN/Instituto Municipal de Planejamento Urbano - IMPLURB, pela contribuição no conhecimento sobre a expansão urbana de Manaus. À Profa. Dra. Eyde Cristianne Saraiva dos Santos/Universidade Federal do Amazonas/Faculdade de Ciências Agrárias, pelo valioso apoio na revisão das informações.

\section{REFERÊNCIAS}

1. Albuquerque BC, Mutis MCS. A malária no Amazonas. In: Rojas LBI, Toledo LM (coords) Espaço e Doença: um olhar sobre o Amazonas. Fundação Oswaldo Cruz, Rio de Janeiro: II.2.1; II.2.7; II.2.8, 1997.

2. Alecrim WD. Estudo clínico - epidemiológico da malária no Rio Ituxí-Amazonas. Tese de Mestrado em Medicina Tropical. Faculdade de Ciências da Saúde, Universidade de Brasília, Brasília, 1979.

3. Alexandre MAA. Estudo clínico e epidemiológico dos casos graves da malária vivax em pacientes atendidos na Fundação de Medicina Tropical do Amazonas, Brasil. Dissertação de Mestrado em Doenças Tropicais e Infecciosas. Fundação de Medicina Tropical do Amazonas, Universidade do Estado do Amazonas, Manaus, 2004.
4. Ávila SLM. Malária. In: Ferreira AW (ed) Diagnóstico laboratorial das principais doenças infecciosas e auto-imunes. Editora Guanabara Koogan, Rio de Janeiro, p. 157-158, 1996.

5. Barata RCB. Malária no Brasil: panorama epidemiológico na última década. Cadernos de Saúde Pública 11: 128-136, 1995.

6. Camargo EP. A malária encenada no grande teatro social. Estudos Avançados. Doença e Sociedade 9: 3-9, 1995.

7. Ferreira MS. Malária. In: Veronesi R, Focaccia R (eds) Veronesi: Tratado de Infectologia. Editora Atheneu, São Paulo, p. 1280 - 1309, 2004.

8. Forattini OP. Culicidologia médica. Editora da Universidade de São Paulo, São Paulo, Volume 2, p.383-390, 2003.

9. Fundação Nacional de Saúde. Controle da malária. Diretrizes técnicas. Ministério da Saúde. Brasília, 1996.

10. Fundação Nacional de Saúde. Guia de Vigilância Epidemiológica. $5^{\mathrm{a}}$ edição Ministério da Saúde. Brasília, p. 559, 2002.

11. Motta EGF. Fatores determinantes da situação da malária na Amazônia. $3^{\mathrm{a}}$ Reunião Nacional dos Pesquisadores em Malária. A pesquisa da malária no Brasil. Revista da Sociedade Brasileira de Medicina Tropical 25 (supl II): 27-28, 1992.

12. Pignatti MG. Saúde e ambiente: as doenças emergentes no Brasil. Ambiente \& sociedade 7: 133-148, 2004.

13. Saraiva MGG. Malária em Manaus: análise epidemiológica, conhecimento e atitudes da população. Dissertação de Mestrado em Doenças Tropicais e Infecciosas. Fundação de Medicina Tropical do Amazonas, Universidade do Estado do Amazonas, Manaus, 2007.

14. Saraiva MGG, Souza RDS, Lopes LDS, Marreiro LS, Amorim RDS. Situação da malária no Estado do Amazonas - 1999 a 2005. Revista da Sociedade Brasileira de Medicina Tropical 39 (supl I): 114, 2006.

15. Secretaria de Vigilância em Saúde. Vigilância Epidemiológica. Malária. Ministério da Saúde, Brasília, 2005a.

16. Secretaria de Vigilância em Saúde. Malária. Guia de Vigilância Epidemiológica. 6a edição. Ministério da Saúde, Brasília, p. 521-531, 2005 b.

17. Secretaria de Vigilância em Saúde. Malária no Brasil, Ministério da Saúde. 2006[?].http://portal.saude.gov.br/portal/arquivos/pdf/be_malaria_01_2005. Acesso em 30.03.2009.

18. Souza JM, Couto AARD, Silva EB, Abdon NP, Silva RSU. Malária. In: Leão RNQ (coord) Doenças Infecciosas e Parasitárias: enfoque amazônico. Editora CEJUP, Belém, PA, p. 645, 1997.

19. Souza SL, Dourado MIC, Noronha CV. Migrações internas e malária urbana Bahia, Brasil. Revista de Saúde Pública 20: 347-351, 1986.

20. Tadei WP, Thatcher BD. Malaria vectors in the Brazilian amazon: Anopheles of the subgenus subgenus Nyssorhynchus. Revista do Instituto de Medicina Tropical de São Paulo 42: 87-94, 2000.

21. Terrazas WCM, Tadei WP, Oliveira AEM, Oliveira AEM, Lopes NR, Queiroz CAB, Figueiredo EO, Filho HPN. Malária em Manaus: situação epidemiológica em 2001 e 2002. Revista da Sociedade Brasileira de Medicina Tropical 36 (supl I): 268, 2003.

22. Terrazas WCM. Desenvolvimento de SIG para análise epidemiológica da distribuição espacial da malária no município de Manaus - um enfoque em nível local. Dissertação de Mestrado em Saúde Pública. Escola Nacional de Saúde Pública, Fundação Oswaldo Cruz, Rio de Janeiro, 2005.

23. Vasconcelos CH, Novo EMLM, Donalisio MR. Uso do sensoriamento remoto para estudar a influência de alterações ambientais na distribuição da malária na Amazônia brasileira. Cadernos de Saúde Pública 22: 3-11, 2006. 